\title{
Hematoma retroperitoneal de causa infrecuente: A propósito de un caso
}

Drs. Daniel Álvarez $\mathrm{G}^{(1)}$, Giancarlo Schiappacasse $\mathrm{F}^{(2)}$, Marcelo Castro $\mathrm{S}^{(3)}$, Jeannie Slater $\mathrm{M}^{(4)}$.

1. Médico General.

2. Departamento de Imaginología, Hospital Militar de Santiago. Clínica Alemana Universidad del Desarrollo

3. Departamento de Imaginología, Hospital Militar de Santiago. Clínica Indisa de Santiago

4. Departamento de Anatomía Patológica, Hospital Militar de Santiago.

Retroperitoneal hematoma of unusual cause: A case report

Abstract: The spontaneous retroperitoneal hematoma (SRH) is defined as a spontaneous hematic collection located in any of the retroperitoneal compartments. The most frequently encountered cause of retroperitoneal hemorrhage is a ruptured abdominal aortic aneurysm, followed by renal and adrenal tumors, along with some other less common causes. Performing accurate diagnosis of patients at admission has proven to be difficult, given that the clinical presentation is ample and variable and may mimic other pictures of abdominal or lower back pain. Imaging techniques, mainly angiography, computed tomography, and MR Imaging, represent a fundamental tool for both timely diagnosis and proper management of the condition. We report the case of a 56-year-old male patient with diagnosis of HIV, who presented with a history of abdominal pain and palpable abdominal mass. After a thorough imaging evaluation, patient was diagnosed with retroperitoneal hematoma, whose histological analysis revealed a Burkitt lymphoma.

Keywords: Burkitt lymphoma, Retroperitoneal hematoma, Spontaneous.

Resumen: El hematoma retroperitoneal espontáneo (HRE) se define como una colección hemática espontánea, situada en cualquiera de los compartimentos retroperitoneales. Dentro de las causas más frecuentes se encuentran las roturas de aneurisma de aorta abdominal, tumores renales y suprarrenales, entre otras. Habitualmente es difícil realizar el diagnóstico al ingreso del paciente, ya que la presentación clínica es variable y puede simular otros cuadros de dolor abdominal o lumbar. Las técnicas de imagen, especialmente la angiografía, tomografía computada y resonancia magnética, son una herramienta fundamental en el diagnóstico y manejo oportuno del cuadro. Presentamos el caso de un varón de 56 años de edad con diagnóstico de VIH en tratamiento, que debutó como un cuadro de dolor y masa abdominal palpable. Luego de un acabado estudio de imágenes, se diagnosticó un HRE cuyo estudio histológico reveló un Linfoma de Burkitt.

Palabras clave: Espontáneo, Hematoma retroperitoneal, Linfoma de Burkitt.

Álvarez D, et al. Hematoma retroperitoneal de causa infrecuente: A propósito de un caso. Rev Chil Radiol 2012; 18(3): 107-110.

Correspondencia: Dr. Daniel Álvarez G. / dunryag@hotmail.com

Trabajo enviado el 17 de julio de 2012. Aceptado el 02 de septiembre de 2012.

Introducción

El hematoma retroperitoneal espontáneo (HRE) corresponde a una colección hemática situada en cualquiera de los 3 compartimentos retroperitoneales: pararrenal anterior, perirrenal y pararrenal posterior. Se produce sin que exista traumatismo, manipulación endourológica o endovascular previa de los elementos del retroperitoneo ${ }^{(1)}$. En la literatura no se han descrito diferencias entre el HRE y la hemorragia retroperitoneal espontánea, por lo que se las considerará sinónimos para fines de esta revisión. Es un cuadro clínico de difícil diagnóstico, que inicialmente puede ser oligosintomático ${ }^{(1)}$, sin embargo, su detección oportuna es fundamental, ya que el retraso diagnóstico determina una alta morbi-mortalidad.
La etiología más frecuente de hematoma retroperitoneal espontáneo es la rotura de un aneurisma de la aorta abdominal. Otras causas de menor frecuencia son: hemorragias espontáneas de tumores renales o suprarrenales, otros tumores sólidos retroperitoneales, terapia anticoagulante, discrasias sanguíneas y patología litiásica de la vía urinaria, entre otros ${ }^{(1,2)}$. Además, se describe asociación con hipertensión arterial y arterioloesclerosis ${ }^{(3)}$.

La presentación clínica del HRE se caracteriza por masa abdominal palpable, dolor lumboabdominal, hipotensión y caída del hematocrito ${ }^{(1,3)}$. Las características e intensidad del dolor son variables y dependen, entre otros factores, de la localización y magnitud del hematoma. Se ha descrito irradiación del dolor hacia 
la región del muslo, cadera y flancos ${ }^{(1-6)}$.

Dada su baja incidencia y manifestaciones clínicas inespecíficas, el diagnóstico clínico de HRE habitualmente es muy difícil. La historia y examen físico deben complementarse con un adecuado estudio imaginológico ${ }^{(1)}$, que habitualmente hace el diagnóstico. Sin embargo, ocasionalmente éste sólo se realiza en el intraoperatorio de forma incidental ${ }^{(3,7)}$.

\section{Caso clínico}

Paciente de sexo masculino de 56 años de edad, portador del virus de inmunodepresión humana $(\mathrm{VIH})$ hace 6 años, actualmente en terapia anti-retroviral (TARV).

Consulta por cuadro de 12 horas de dolor en fosa lumbar izquierda, de aparición brusca, tipo cólico, asociado a náuseas. Presenta respuesta parcial a analgesia AINE y antieméticos. TC no contrastada (PieloTC) revela hematoma retroperitoneal izquierdo de $16 \times 12 \times 8 \mathrm{~cm}$ en los ejes céfalo-caudal, coronal y ántero-posterior. Evoluciona con shock hipovolémico, alcanzando un hematocrito de $29 \%$ por lo que recibe transfusión de 2 unidades de glóbulos rojos. Se realiza angiografía selectiva que descarta hemorragia activa y vasos de neoformación.

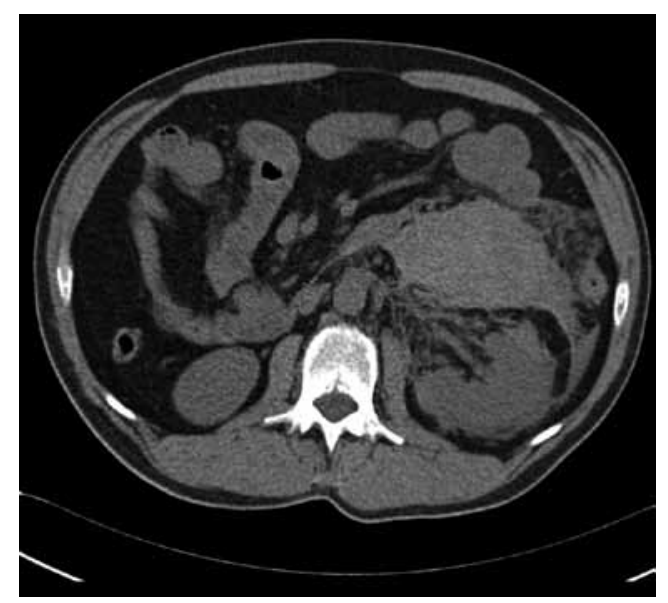

Figura 1. TC de abdomen y pelvis al ingreso del paciente. TC de abdomen y pelvis no contrastado. Corte axial muestra extenso hematoma retroperitoneal izquierdo ubicado preferentemente en el espacio pararrenal anterior. El riñón se observa respetado.

Presenta durante los días siguientes un cuadro febril que se atribuye a reabsorción de su hematoma. Dentro de exámenes de laboratorio destaca carga viral indetectable para $\mathrm{VIH}$, recuento de CD4 mayor a 500 células/uL, TP de 13.2 segundos de y TTPK de 30 segundos.

Por persistencia del dolor lumbar, sin variación en su Hcto., que era de $35 \%$, se realiza TC una semana más tarde, la cual revela leve crecimiento del hematoma retroperitoneal, permaneciendo con parámetros hemodinámicos estables, por lo que se da de alta con indicación de control en una semana.

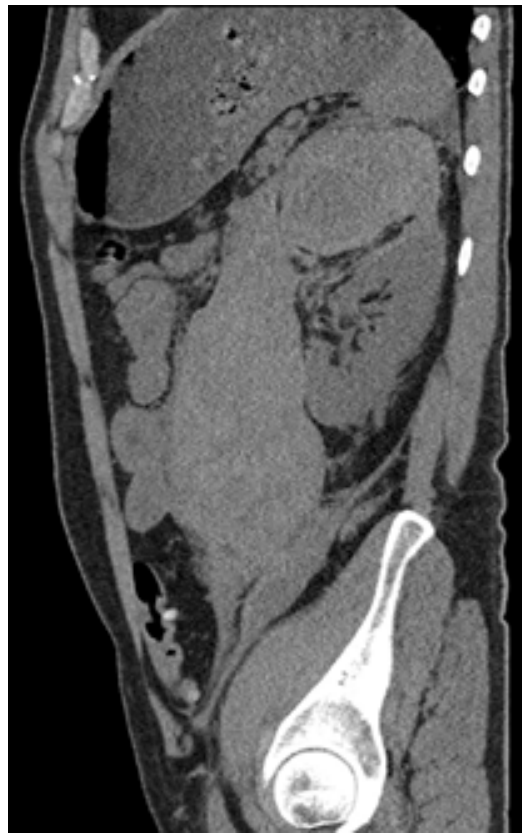

Figura 2. TC de abdomen y pelvis con contraste en fase arterial. Corte axial y sagital. Respecto al examen anterior, no se observan zonas de impregnación con contraste en la masa retroperitoneal.

En siguiente TC, efectuado a 2 semanas de su ingreso, se observa regresión parcial del hematoma, el que además presenta cambios de su estructura caracterizados por disminución de su tamaño céfalocaudal y aparición de algunas imágenes nodulares densas.

Se efectúa RM de abdomen que confirma una masa retroperitoneal mixta con zonas hemorrágicas antiguas y un componente sólido periférico y nodular, pobremente vascularizado.

Posteriormente se realiza biopsia percutánea guiada por TC, cuyo análisis histopatológico revela linfoma no Hodgkin difuso de células medianas. Se decide tratamiento en Unidad de Quimioterapia con esquema R-CHOP.

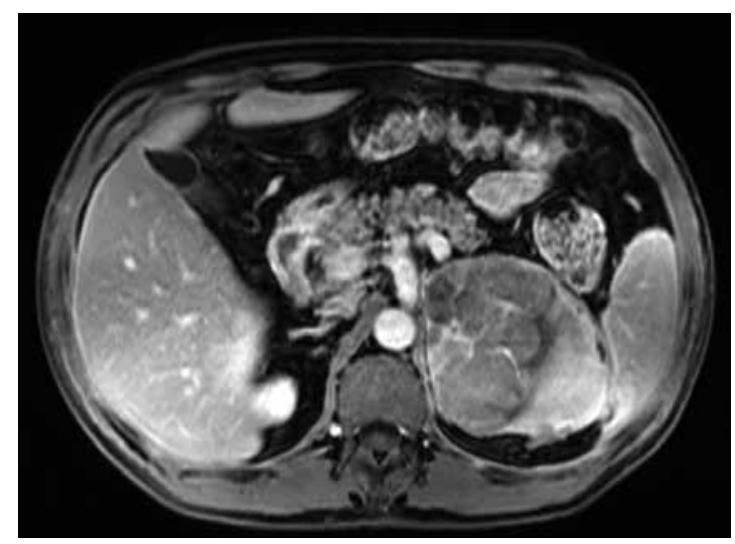

Figura 3. RM de control. Corte axial en T1, supresión grasa y uso de gadolinio. Se observa leve aumento de señal correspondiente al componente sólido de la masa. 


\section{Discusión}

El linfoma de Burkitt (LB) es un tipo infrecuente de linfoma no Hodgkin. Puede afectar tanto a niños como a jóvenes, sin embargo se ha descrito un promedio de edad mayor a 40 años al momento de su diagnóstico ${ }^{(8)}$. Clínicamente, es una enfermedad agresiva que con frecuencia compromete tejido linfático extranodal ${ }^{(9)}$.

Se reconocen tres tipos bien de LB: endémico, esporádico y asociado a inmunodeficiencia. Los tres tipos se caracterizan por la presencia de translocaciones cromosomales que involucran 8q24/MYC, lo que lleva a una disrregulación MYC por yuxtaposición con genes regulatorios de la expresión de inmunoglobulinas $^{(9)}$.

El LB esporádico es la forma de presentación más frecuente de este grupo de linfomas. Representa entre 1 y $2 \%$ del total de los LNH en USA y Europa ${ }^{(9)}$. Clínicamente, predominan los síntomas gastrointestinales debido a su alta frecuencia de compromiso ileocecal; aunque también puede afectar al sistema nervioso central (SNC), médula ósea (MO) y tórax

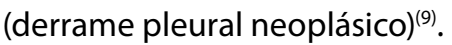

Típicamente, los pacientes se presentan con una masa abdominal palpable, síntomas B y evidencia de lisis tumoral en exámenes de laboratorio complementarios $^{(8)}$.

EI LB endémico se observa principalmente en África ecuatorial y ahí corresponde al tumor infantil más común. Se presenta principalmente en huesos del macizo facial, y suele existir evidencia de infección previa por virus Epstein-Barr además de alta correlación geográfica con Malaria endémica ${ }^{(9)}$.

EI LB asociado a inmunodepresión se observa principalmente en pacientes con SIDA, pero también se puede observar en pacientes inmunosuprimidos farmacológicamente después de un trasplante de médula ósea u órgano sólido. El compromiso nodal es la forma más frecuente de presentación, pero también se puede observar compromiso de la MO y el SNC.

El diagnóstico de LB se fundamenta en el estudio histopatológico tradicional, complementado con técnicas inmunofenotípicas, inmunogenéticas, análisis de genética molecular y datos clínicos ${ }^{(10)}$. El tumor está compuesto por células linfoides monomorfas de tamaño mediano, con alta tasa mitótica y alto índice de proliferación Ki67. Es característica una translocación entre los genes MYC e IG ${ }^{(11)}$.

El caso presentado corresponde a una forma de presentación atípica de LB. La información clínica es relevante, ya que el paciente presentaba el antecedente de inmunodepresión secundaria a VIH, que es el contexto clínico más frecuente de presentación de esta forma de LB. A pesar de lo anterior, este dato clínico sólo contribuyó al diagnóstico en forma retrospectiva, apoyándolo, ya que después de reiterados estudios de imagen se planteó linfoma como diagnóstico

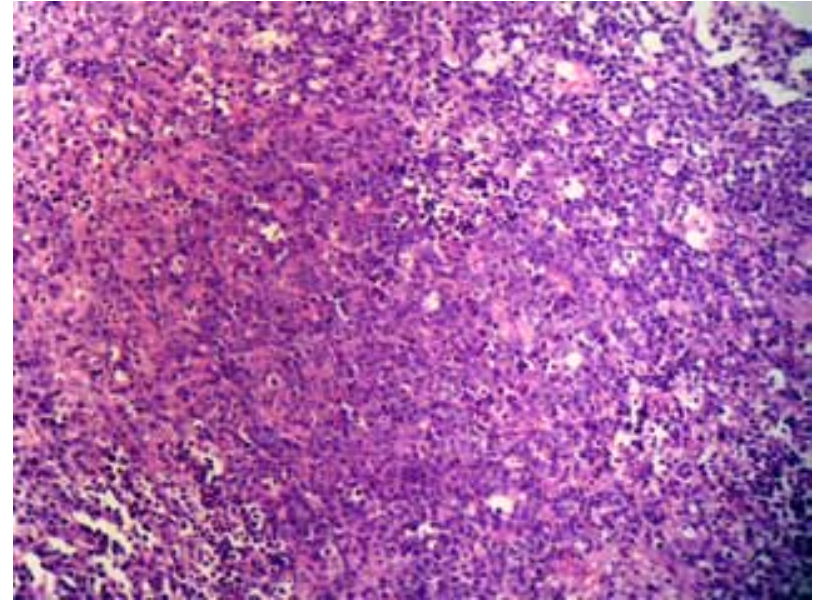

Figura 4. Histología HE, CD20, Ki67.

diferencial, lo que motivó al estudio histológico que finalmente hizo el diagnóstico específico. No existen en la literatura reportes de casos de LB que hayan debutado de esta manera, por lo que debe considerarse un aporte en el planteamiento diagnóstico del hematoma retroperitoneal espontáneo, especialmente en el contexto del paciente inmunodeprimido.

Por otro lado, es importante resaltar el aporte de la RM que gracias a su mejor resolución de contraste, permitió diferenciar el tejido tumoral del componente hemático, lo que permitió finalmente hacer el diagnóstico. En último término, cabe mencionar que el estudio angiográfico realizado para pesquisar y eventualmente tratar el sitio de la hemorragia no demostró vasos de neoformación, situación no infrecuente en masas tumorales linfomatosas, a diferencia de otros tumores retroperitoneales.

\section{Bibliografía}

1. Gimeno Argente V, Bosquet Sanz M, Ramírez Backhaus $M$, Trassierra Villa $M$, Arlandis Guzmán S, Jiménez Cruz JF. Hemorragia retroperitoneal espontánea: nuestra experiencia en los últimos 10 años. Actas Urológicas Españolas, 2007; 31(5): 521-527.

2. Moazzam M, Hammad Ather M, Hussainy A. Leiomyosarcoma presenting as a spontaneously ruptured renal tumor-case report. BMC Urology, 2002; 2: 13.

3. Álvarez C, Cerda C, Jadue A, Rojas F, Abelleira $M$, Hermansen $C$, et al. Hematoma retroperitoneal espontáneo. Caso clínico. Revista Médica de Chile, 2007; 135: 1044-1047.

4. Sun P, Lee Y, Chiu K. Retroperitoneal hemorrhage caused by enoxaparin-inducedspontaneous lumbar artery bleeding and treated by transcatheter arterial embolization: a case report. Cases Journal 2009; 2: 9375.

5. Daliakopoulos S, Bairaktaris A, Papadimitriou D, Pappas P. Gigantic retroperitoneal hematoma as a complication of anticoagulation therapy with heparin in therapeutic doses: a case report. Journal of Medical Case Reports, 2008; 2: 162.

6. Hanna W, Myles J. Spontaneous Intraperitoneal Hae- 
morrhage During Pregnancy: Report of Three Cases. Brittish Medical Journal 1964; 1(5389):1024-1026.

7. Maxit M, Paz R. Causas inusuales de hematoma perirrenal y retroperitoneal. Revista del Hospital Privado de Comunidad, 2000; : 18-22.

8. Perkins A, Friedberg J. Burkitt Lymphoma in Adults. Hematology, 2008; 1: 341-348.

9. Sweetenham J. Highly Aggressive Lymphomas in Adults. Hematology Oncology Clinics of North
America, 2008; 22(5): 965-978.

10. Holte H, Kvaløy S, Delabie J, Trøen G, Smeland EB. Tidsskrift for den Norske Laegeforening. Molecular diagnosis of malignant lymphomas. 2009; 129(22): 2352-2356.

11. De Leval L, Hasserjian R. Diffuse Large B-Cell Lymphomas and Burkitt Lymphoma. Hematology Oncology Clinics of North America, 2009; 23(4): 791-827. 